\title{
Notes on black holes and three dimensional gravity
}

\author{
Máximo Bañados \\ Departamento de Física Teórica, Universidad de Zaragoza, Ciudad Universitaria 50009, \\ Zaragoza, Spain.
}

\begin{abstract}
.
These notes are the written version of two lectures delivered at the VIII Mexican School on Particles and Fields on November 1998. The level of the notes is basic assuming only some knowledge on Statistical Mechanics, General Relativity and YangMills theory. After a brief introduction to the classical and semiclassical aspects of black holes, we review some relevant results on $2+1$ quantum gravity. These include the Chern-Simons formulation and its affine Kac-Moody algebra, the asymptotic algebra of Brown and Henneaux, and the statistical mechanics description of $2+1$ black holes. Hopefully, this contribution will be complementary with the review paper hepth/9901148 by the same author, and perhaps, a shortcut to some recent developments in three dimensional gravity.
\end{abstract}

\section{INTRODUCTION}

During the last three years we have witnessed a rapid progress in the string theory description of general relativity. Successfull computations of black hole entropy for extremal and near extremal solutions $[1,2]$ have made it clear that the string theory degrees of freedom describes the expected semiclassical behaviour of general relativity. This is in sharp constrast with the more standard approach to quantum gravity either based on the path integral approach or the Wheeler-de Witt equation which has provided little information about the fundamental degrees of freedom giving rise to the Bekenstein-Hawking entropy. In the Loop representation approach to quantum gravity, a computation of the black hole entropy has been proposed [3,4]. However, in this formulation it is still obscure how to introduce dynamics, and only the kinematics of spin networks is under control.

In this contribution we shall consider neither string theory nor loop quantum gravity. Instead, we work in the very simple setting of three-dimensional quantum gravity whose Lagrangian describes a well-defined quantum field theory [5,6]. As motivations to study three-dimensional gravity, let us mention the following aspects of it. (i) It is a mathematically simple theory which combines three important branches of physics: General Relativity, Yang-Mills theory (with a Chern-Simons 
action), and two-dimensional Conformal Field Theory. (ii) The mathematical tools are surprinsingly similar to those used in string theory, with a centrally extended Virasoro algebra [7] as one of its main ingredients. (iii) The space of solutions contains particle solutions [8] and black holes [9], thus making it interesting from the dynamical point of view.

In these notes, we shall mainly be interested in quantum black holes in three dimensions. Our goal is to give, in a somehow self-contained way, a derivation of Strominger's [10] proposal for the statistical mechanical origin of the three-dimensional black hole entropy. We refer the reader to [11-14] for the stringy aspects of Strominger's result. See also [15] for a recent review. We shall concentrate here on the gravitational aspects. For a detailed and complete treatment of three dimensional gravity we refer to the recent book by Carlip [16].

In Sec. II we shall briefly review, at the most basic level, some of the main properties of the Schwarzschild solution, as well as the three-dimensional black hole [9]. In Sec. III we review the Chern-Simons formulation of three-dimensional gravity. Particular empahsis is given to the absence of bulk degrees of freedom, and a quick derivation of the affine Kac-Moody algebra is presented. Finally, in Sec. IV we derive the Brown-Henneaux conformal algebra, and its statistical mechanical $[10,45]$ implications.

\section{CLASSICAL AND SEMICLASSICAL BLACK HOLES}

\section{A The black hole spacetime}

The Schwarzschild metric $\left(r>r_{0}\right)$,

$$
d s^{2}=-(1-2 M / r) d t^{2}+(1-2 M / r)^{-1} d r^{2}+r^{2} d \Omega^{2},
$$

is an exact solution of the Einstein vacuum equations

$$
G_{\mu \nu}=R_{\mu \nu}-\frac{1}{2} g_{\mu \nu} R=0,
$$

representing the geometry outside a collapsing star of mass $M$ and radius $r_{0}$. One of the most surprising predictions of General Relativity, which caused much confusion in the past, is the appearance of a singularity in the metric for the particular value of $r$ :

$$
r=: r_{+}=2 M \quad \text { (Event Horizon). }
$$

If the radius of the star $r_{0}$ is less that $r_{+}$then the solution (1), which is valid for $r>r_{0}$, has a singularity at $r=r_{+}$. Furthermore, in the region $0<r<r_{+}$where the metric is again regular, $r$ is a timelike coordinate while $t$ is spacelike. Finally, at $r=0$ the curvature blows up making gravitational forces divergent there. This means, in particular, that no observer can reach the singularity without being 
destroyed. The possibility of making experiments near the singularity is prevented by another fact: any observer that crosses the event horizon $r=r_{+}$will never come back, at least not according to the classical Einstein equations. We shall prove this below. Quantum mechanically, particles can tunnel out of the black hole and escape to infinity. This is Hawking's famous discovery of black hole radiation [26]. However, according to Hawking's description there is no correlation between the particles that fall into the black hole with the ones that escape. This point is actually a matter of discussion and there is no agreement yet. We will not have time here to describe in any detail this very interesting work. We refer the interested reader, for example, to [24] for a review with an extensive list of references.

Let us now briefly show how to deal with the $r=r_{+}$singularity in (1). This will allow us to see why observers cannot travel back once they have crossed the horizon. We shall also infer the value of Hawking's temperature via a geometrical argument.

The analysis that follows does not depend on the details of the Schwarzschild solution but only on some general properties of black holes. We consider general metrics in $d$ dimensions of the form,

$$
d s^{2}=-f(r) d t^{2}+f^{-1}(r) d r^{2}+d s_{d-2}^{2},
$$

where $d s_{d-2}^{2}$ represents the metric of a $S_{d-2}$ sphere, or some other compact or noncompact surface. The function $f(r)$ satisfies the following two properties: (i) There exists a value of $r$ denoted as $r_{+}$such that $f\left(r_{+}\right)=0$; (ii) The derivative of $f$ at $r_{+}$is different from zero,

$$
\left.\alpha \equiv \frac{1}{2} \frac{d f(r)}{d r}\right|_{r=r_{+}}, \quad \alpha \neq 0 .
$$

Most known (non-extreme!) black holes have a metric of this form, or at least there is a plane on which the metric near $r=r_{+}$looks like the first two terms in (4). The extreme black holes do not fall into the above class of metrics because the function $f(r)$ has a second order zero and thus $\alpha=0$. These black holes play an important role in string theory because they are related to BPS states.

For the Schwarzschild black hole, the function $f$ is given by $f(r)=1-2 M / r$ and $f^{\prime}\left(r_{+}\right)=1 / 2 M$. This means that $\alpha=1 / 4 M$ which is indeed different from zero. Other examples are: The Reissner-Nordstrom black hole with $f(r)=1-2 M / r+$ $e^{2} / r^{2}$ and $e$ is the electric charge. In this case, $\alpha$ is different from zero provided $M \neq e$; The $2+1$ black hole (to be studied in detail in the next section) with $f(r)=-M+r^{2} / l^{2}$ and $l$ is related to a cosmological constant; The d-dimensional Schwarzschild solution with $f(r)=1-2 M / r^{d-3}$; plus all the (non-extreme) stringy black holes [27], as well as other higher dimensional situations [28]. Students are encouraged to compute the value of $\alpha$ for each of these black holes, as we shall see soon, this number is essentially Hawking's temperature for each of these objects.

The metric (4) is singular at the event horizon, just as the Schwarzschild metric is. To cure this singularity we introduce the following new set of coordinates. We change $\{r, t\}$ to $\{u, v\}$ according to, 


$$
\begin{aligned}
& u=g(r) \cosh \alpha t, \\
& v=g(r) \sinh \alpha t,
\end{aligned}
$$

where the function $g(r)$ is defined by,

$$
g(r)=\exp \left(\alpha \int^{r} \frac{d r^{\prime}}{f\left(r^{\prime}\right)}\right) .
$$

This change of coordinates has the following properties. The event horizon $r=r_{+}$ is mapped into the lines $u= \pm v$. The metric in terms of $u, v$ reads,

$$
d s^{2}=\Omega^{2}(r)\left(-d v^{2}+d u^{2}\right)+r^{2} d \Omega^{2},
$$

where the function,

$$
\Omega^{2}(r)=\frac{f(r)}{\alpha^{2} g^{2}(r)},
$$

is regular at $r=r_{+}$. The regularity of $\Omega^{2}$ holds provided $f(r)$ has a simple pole at $r=r_{+}$. It is easy to see using L'Hopital rule that the zero in $f(r)$ is cancelled by $g^{2}(r)$ provided $\alpha$ is chosen as in (5). Note that the above coordinate change does not depend on the details of the function $f(r)$, provided it has a single pole at $r=r_{+}$. Of course, our formulae for the conformal factor and change of coordinates reproduce the usual expressions when applied to particular situations like the Schwarzschild black hole (see [25]).

The coordinates $u$ and $v$ are called Kruskal coordinates and their range is $-\infty<$ $u, v<\infty$. These coordinates can be compactified (see [25] for more details on this points) and led to the Penrose diagram shown in Fig. $1^{1}$. Region $\mathbf{I}$ is the black hole exterior $r>r_{+}$and region II its interior $\left(r<r_{+}\right)$. It should be clear from the figure that an observer situated in region II cannot go back to region $\mathbf{I}$ because he or she would need to travel faster than light. The fate of any future-directed (timelike) observer is to hit the singularity.

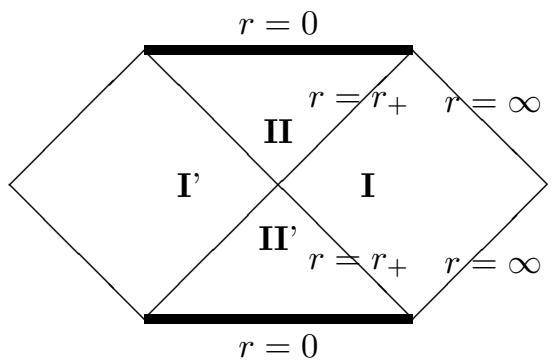

1

Fig.

Penrose diagram for a Schwarzschild black hole.

1) The Penrose diagram shown here assumes asymptotic flatness. This means that the function $f(r)$ satisfies $f(r \rightarrow \infty) \rightarrow 1$. This is not the case for asymptotically anti-de Sitter black holes for which $f \rightarrow r^{2}$. The Penrose diagram in that case can still be drawn and differs only in the asymptotic structure, not the properties surrounding the horizon. See second Ref. in [9]. 


\section{B Semiclassical black holes. The Gibbons-Hawking approximation}

The combination of Euclidean field theory together with the coordinate change (6) suggest in a very direct way that black holes should have a non-zero temperature. The Euclidean formalism (sometimes called the Euclidean sector) is obtained by setting $\tau=i t$, and the metric (1) becomes Euclidean. Consider again the change of coordinates (6) in the Euclidean formalism. The hyperbolic functions will be replaced by their trigonometrical versions and it is clear that the Euclidean time variable needs to be an angle $0 \leq \alpha \tau<2 \pi$. In the Euclidean sector, and near the horizon, the change (6) is nothing but the relation between polar and cartesian coodinates in $\Re^{2}$. In fact, the topology of the Euclidean Schwarzschild black hole is $\Re^{2} \times S_{2}$ where the origin of $\Re^{2}$ is situated at the horizon $r=r_{+}$. The Euclidean sector does not see the inner region of the black hole $r<r_{+}$.

Following the usual practice of Euclidean field theory we define the inverse temperature as the Euclidean time period $(\hbar=1)$,

$$
\beta=\frac{2 \pi}{\alpha}
$$

So far this is only a mathematical trick with no real physics meaning. However, it turns out that the temperature $T=1 / \beta$ defined in (10) coincides exactly with Hawking's evaporation temperature. For the Schwarzschild black hole, we recall that $\alpha=1 / 4 M$, this yields the famous Hawking result,

$$
T_{H}=\frac{1}{\beta_{H}}=\frac{1}{8 \pi M}
$$

Now, integrating the first law $d M=T d S$ we find the Bekenstein-Hawking formula for black hole entropy,

$$
S=\frac{A}{4}
$$

where $A=4 \pi r_{+}^{2}$ is the area of the event horizon $\left(r_{+}=2 M\right)$.

For our purposes, this "derivation" of the black hole temperature and entropy has an important meaning: geometry knows that black holes radiates. In other words, the very deep origin of Hawking's process is not contained only on the matter fields surrounding a black hole but rather on the gravitational (perhaps string) degrees of freedom. This point of view is further supported by the GibbonsHawking calculation of the Schwarzschild black hole partition function which we now describe.

Let us briefly review here the results presented in [17] in the simplest case of a non-rotating black hole. Our main tool will be again the analogy between Euclidean field theory and statistical mechanics.

Consider the functional integral, 


$$
Z[h]=\int D g e^{-I[g, h]},
$$

where,

$$
I[g, h]=-\frac{1}{16 \pi G}\left(\int_{M} \sqrt{g} R+2 \int_{\partial M} \sqrt{h} K\right) .
$$

is the Euclidean gravitational action appropriated to fix the metric at the boundary. Here $h_{i j}$ is the 3 -metric induced on $\partial M$. The boundary term is added to the action to ensure that $I$ has an extremum when $h$ is fixed. $D g$ denotes the sum, modulo diffeomorphisms, over all metrics with $h_{i j}$ fixed. As it is well known, the formula (13) is purely formal and cannot be given a precise mathematical meaning. This is because gravity is not renormalizable and the perturbation expansion for (13) is not well-defined. To make things worst, the action (14) is not bounded from below/above, not even in the Euclidean formulation. It is possible to find sequences of Euclidean manifolds $M_{i}$ for which the value of the action (14) goes to minus/plus infinity [18].

Although (13) cannot be computed in general, its saddle point approximation around some classical solutions gives interesting results. Incidentally, we mention here that the evaluation of the action $I$ on classical solutions has become crucial in the recently discovered adS/CFT correspondence [19-21]. The first example of an evaluation of (13) was performed by Gibbons and Hawking [17] who considered the Euclidean Schwarzschild black hole (1) with mass $M$. The mass $M$ and Euclidean period $\beta$ are related by (11) in order to avoid singularities (sources) in the Euclidean metric. The value ${ }^{2}$ of $Z$ in the saddle point (1) is

$$
Z[\beta] \sim e^{-\beta^{2} / 16 \pi} .
$$

This result is quite remarkable. The thermodynamical formula for the average energy $M=-\partial \log Z / \partial \beta$ reproduces (11) and confirms that $\beta$ is the inverse temperature of the black hole. In the same way, the average entropy $S=\ln Z-\beta \partial_{\beta} \ln Z$ reproduces (12). This result confirms once again that the black hole thermal properties are present in a pure quantum theory of gravity, and not only in the interaction of a classical background metric with quantized fields.

Hawking's discovery of black hole evaporation is one of the most important results in the theory of general relativity and quantum mechanics. We refer the reader, for example, to the classic books by Birrell and Davis [29] and Wald [30] for a detailed discussion on quantum black holes, and in particular, quantum field theory on curved spacetimes. These books were written before black holes became important in string theory. See [31] for a review on the string theory approach to black holes.

We shall now depart from the Schwarzschild four dimensional black hole and go down to three dimensions where a black hole solution exists [9] having many of the features of the Schwarzschild solution, but it is far simpler mathematically.

2) Actually, the value of $I$ diverges and needs to be regularized. See [17] for details and $[33,34]$ for an evaluation of $I$ using Hamiltonian methods on which the regularization is automatic. 
Consider the action for three-dimensional gravity with a negative cosmological constant $\Lambda=-2 / l^{2}$

$$
I=\frac{1}{16 \pi G} \int \sqrt{-g}\left(R+\frac{2}{l^{2}}\right) d x^{3} .
$$

In three dimensions it is convenient to keep the fundamental constants because, due to the cosmological constant, there are two fundamental length parameters: Plank's length $l_{p}=\hbar G$ and the cosmological radius $l$.

The equations of motion derived from this action are solved by the (non-rotating) three-dimensional black hole [9]

$$
d s^{2}=-\left(-8 M G+\frac{r^{2}}{l^{2}}\right) d t^{2}+\left(-8 M G+\frac{r^{2}}{l^{2}}\right)^{-1} d r^{2}+r^{2} d \varphi^{2} .
$$

Angular momentum as well as electric charge can be added easily, see [9].

As for the Schwarzschild metric, we can go to the Euclidean sector and discover that the time coordinate is periodic. The associated temperature is,

$$
T_{3}=\frac{\sqrt{M}}{2 \pi l^{2}}
$$

and the entropy is again given by (12), but now $A=2 \pi r_{+}$is the perimeter length of the horizon.

A word of caution is necessary here. Contrary to the Schwarzschild case, the metric (17) is not asymptotically flat. This means that the Euclidean period cannot be defined as the proper length of the time coordinate at infinity. Note that the limit $r \rightarrow \infty$ of the Euclidean Schwarzschild metric yields a well defined metric at the "boundary" (infinity is not really a boundary) with the topology $S_{1} \times S_{2}$. $S_{1}$ corresponds to the periodic time coordinate, while $S_{2}$ to the angular sphere. The limit $r \rightarrow \infty$ of (17) is not well defined. At infinity, one can only define a conformal class of metrics . This is a three-dimensional example of the adS/CFT correspondence [19-21] first studied in [7]. A more rigorous definition for the temperature can be given by noticing that the topology of (17) in the Euclidean sector is a solid torus. The temperature is related to the complex structure of the torus $[37,38]$ by,

$$
\tau=\frac{\beta}{2 \pi}\left(\Omega+\frac{i}{l}\right)
$$

where $\beta$ is the period of the Euclidean time coordinate, and $\Omega$ is the angular velocity. In the non-rotating case, $\Omega=0$.

As before, one can write down the three-dimensional partition function in the saddle point approximation provided by the solution (17). This yields [9] (see also [52] for a Lagrangian approach),

$$
Z_{3} \sim e^{\pi^{2} l^{2} /(2 G \beta)}
$$


and it is direct to check that the thermodynamical formulae for the average energy and entropy is consistent with (18).

Our main motivation to study three dimensional quantum gravity is to try to give a precise meaning to the formula (13) in three dimensions. In other words, we hope that in three dimensions (13) could be well-defined mathematically, and provide the semiclassical limit (20). If this is true, then one should be able to extract from the exact formula for $Z$ which are the degrees of freedom giving rise to the black hole entropy.

The main mathematical device that we shall use is the Chern-Simons formulation of three dimensional gravity $[5,6]$. This formulation makes manifest the fact that three dimensional gravity does not have any bulk degrees of freedom and it is renormalizable. Still, this does not mean that the problem is trivial because the relevant group, see below, is $S L(2, C)$ which is not compact. The evaluation of the partition function for the black hole problem using the Chern-Simons formulation was initiated by Carlip [44]. Some clarification on the boundary conditions and the role of ensembles can be found in [37]. A string theory approach can be found in [38]. Further developments on the modular properties of the partition function in three dimensions have recently appeared in [39].

\section{2+1 GRAVITY AS A CHERN-SIMONS THEORY.}

\section{A First order form of the Euclidean action}

We start with the (Palatini) Euclidean action for three dimensional gravity with a negative cosmological constant $\Lambda=-2 / l^{2}$,

$$
I\left[g_{\mu \nu}, \Gamma_{\mu \nu}^{\rho}\right]=\frac{1}{16 \pi G} \int \sqrt{g}\left(g^{\mu \nu} R_{\mu \nu}(\Gamma)+\frac{2}{l^{2}}\right)
$$

The discovery of Achúcarro and Townsend is that in three dimensions one can replace the metric by two Yang-Mills fields such that both the structure of the action and equations of motion simplifies enormously.

This is achieved in various steps. First, we use the Palatini formalism. The idea of this formalism is to note that the Ricci tensor $R_{\mu \nu}$ depends on the metric only through the Christoffel symbol $\Gamma_{\mu \nu}^{\rho}$. Then, it follows that if one treats $g_{\mu \nu}$ and $\Gamma_{\mu \nu}^{\rho}$ as independent variables in the action (21), the equations of motion yield the expected relation $g_{\mu \nu ; \lambda}=0$ between the metric and connection. Next, we make a change of coordinates from the coordinate basis $\partial_{\mu}$ to orthonormal coordinates on which the metric is flat. The matrix that makes this change is called the triad and is defined by the formula,

$$
g_{\mu \nu}=e_{\mu}^{a} \eta_{a b} e_{\nu}^{b}
$$

Clearly, $e_{\mu}^{a}$ is defined only up to a (local) Lorentz rotation because if $\Lambda$ is an element of the Lorentz group then, by definition, $\Lambda \eta \Lambda^{-1}=\eta$. Equation (22) is nothing but 
the transformation of a tensor under a change of coordinates described by the matrix $e_{\mu}^{a}$. We also need to transform the Christoffel symbol which is not a tensor but we know its transformation law under $e_{\mu}^{a}$,

$$
\Gamma_{\mu \nu}^{\sigma}=e_{a}^{\sigma} \omega_{b \nu}^{a} e_{\mu}^{b}+e_{a}^{\sigma} e_{\mu, \nu}^{a}
$$

where $w_{\mu}^{a b}$, known for historical reasons as the spin connection, is the new 'Christoffel symbol' in the new coordinates. Eq. (23) is often written in the literature as $e_{\mu ; \nu}^{a}=0$ where the semicolon denoted full covariant derivative, or as $D_{\mu} e_{\nu}^{a}=\Gamma_{\nu \mu}^{\rho} e_{\rho}^{a}$ where $D_{\mu}$ denotes covariant derivative in the spin connection. These formulae are, of course, equivalent to (23). Note that in (23) we have only transform two indices. The reason is that the Christoffel symbol is a 1-form connection for the group $G L(4, \Re), \Gamma_{\nu}^{\mu}=\Gamma_{\nu \rho}^{\mu} d x^{\rho}$. The next object we would like to write in the new coordinates is the curvature tensor. The curvature tensor is a tensorial 2-form, for that reason we only transform two of its four indices as,

$$
R_{\mu \nu}^{\lambda \sigma}=e_{a}^{\lambda} e_{b}^{\sigma} R_{\mu \nu}^{a b}
$$

where $R^{a b}=d w^{a b}+w_{{ }^{\wedge}}^{a} w^{c b}$. With formulae (22), (23) and (24) at hand we can prove the identity

$$
\int \epsilon_{a b c} R^{a b} \wedge e^{c}=\int \sqrt{g} R
$$

The relevant steps are (we go from left to right),

$$
\begin{aligned}
\int \epsilon_{a b c} R^{a b}{ }_{\wedge} e^{c} & =\int \epsilon^{\mu \nu \lambda} \epsilon_{a b c}\left(\frac{1}{2} R_{\mu \nu}^{a b}\right) e_{\lambda}^{c} \\
& =\frac{1}{2} \int \epsilon^{\mu \nu \lambda} \epsilon_{a b c} R_{\mu \nu}^{\alpha \beta} e_{\alpha}^{a} e_{\beta}^{b} e_{\lambda}^{c} \\
& =\frac{1}{2} \int \epsilon^{\mu \nu \lambda} \epsilon_{\alpha \beta \lambda} e R_{\mu \nu}^{\alpha \beta} .
\end{aligned}
$$

In the second line we have used (24), and in the third line $\epsilon_{a b c} e_{\alpha}^{a} e_{\beta}^{b} e_{\lambda}^{c}=e \epsilon_{\alpha \beta \lambda}$ with $e$ equal to the determinant of $e_{\mu}^{a}$, and $\epsilon^{\mu \nu \lambda} \epsilon_{\alpha \beta \lambda}=\delta_{[\alpha \beta]}^{[\mu \nu]}$ (recall that we are working in the Euclidean formalism). It should be clear that the last line in (26) is equal to the right hand side of $(25)$.

Collecting all formulae together we arrive at the new action for three-dimensional gravity,

$$
I\left[e^{a}, w^{a b}\right]=\frac{1}{16 \pi G} \int \epsilon_{a b c}\left(R^{a b}+\frac{1}{3 l^{2}} e^{a} \wedge e^{b}\right) \wedge e^{c} .
$$

The action $I[e, w]$ is equal to the action $I[g, \Gamma]$ shown in (21). Besides notation issues there is a conceptual consequence. The action (27) is perfectly well defined even is the metric is degenerate. In this sense, the triad formulation provides a generalization for the Einstein-Hilbert action.

The last step before we can write the Chern-Simons action is to define the new spin connection ${ }^{3} w^{a}$ and curvature $R^{a}$,

3) With this definition of $w^{a}$ the torsion becomes $T^{a}=d e^{a}+\epsilon_{b c}^{a} w^{b} \wedge e^{c}$. 


$$
w^{a}=-(1 / 2) \epsilon_{b c}^{a} w^{b c}, \quad R^{a}=-(1 / 2) \epsilon_{b c}^{a} R^{b c}
$$

with $R^{a}=d w^{a}+\frac{1}{2} \epsilon_{b c}^{a} w^{b} \wedge w^{c}$.

We are now ready to make the connection with Chern-Simons theory. Let $x$ be a complex number and let $A^{a}$ and $\bar{A}^{a}$ to fields related to $e$ and $w$ by,

$$
A^{a}=w^{a}+x e^{a}, \quad \bar{A}^{a}=w^{a}-x e^{a} .
$$

The relation between Chern-Simons theory and three dimensional general relativity follows from the equality:

$$
\begin{aligned}
2 e_{a} R^{a}+\frac{x^{2}}{3} \epsilon_{a b c} e^{a} e^{b} e^{c}= & \frac{1}{2 x}\left(A_{a} d A^{a}+\frac{1}{3} \epsilon_{a b c} A^{a} A^{b} A^{c}\right) \\
& -\frac{1}{2 x}\left(\bar{A}_{a} d \bar{A}^{a}+\frac{1}{3} \epsilon_{a b c} \bar{A}^{a} \bar{A}^{b} \bar{A}^{c}\right)+d B .
\end{aligned}
$$

This relation is true regardless the signature of spacetime or sign of the cosmological constant. Just plug (29) into the right hand side of (30) and obtain the left hand side. ( $d B$ is a total derivative term.)

Depending on the signature of spacetime and cosmological constant $x$ need to be complex or real. We shall be interested here in the Euclidean gravity with a negative cosmological constant. In the case, $x$ is purely imaginary.

\section{B Chern-Simons action}

From the equality (30) with $x=i / l$ it follows that the Einstein-Hilbert action (21) for Euclidean three dimensional gravity can be written in the form,

$$
I[g, \Gamma]=i I[A]-i I[\bar{A}]
$$

where $I[A]$ is the Chern-Simons action,

$$
I[A]=\frac{k}{4 \pi} \int \operatorname{Tr}\left(A d A+\frac{2}{3} A^{3}\right)
$$

at level ${ }^{4}$,

$$
k=-\frac{l}{4 G}
$$

In (31) we have defined

$$
A=A^{a} J_{a}, \quad \bar{A}=\bar{A}^{a} J_{a} .
$$

4) The sign of $k$ depends on the identity $\sqrt{g}= \pm e$ where $e$ is the determinant of the triad. This sign determines the relative orientation of the coordinate and orthonormal basis. We have chosen here the plus sign which means that we work with $e>0$. 
where the $S U(2)$ generators are given by,

$$
J_{1}=\frac{i}{2}\left(\begin{array}{ll}
0 & 1 \\
1 & 0
\end{array}\right), \quad J_{2}=\frac{1}{2}\left(\begin{array}{cc}
0 & -1 \\
1 & 0
\end{array}\right), \quad 3=\frac{i}{2}\left(\begin{array}{cc}
1 & 0 \\
0 & -1
\end{array}\right),
$$

and satisfy $\left[J_{a}, J_{b}\right]=\epsilon_{a b}{ }^{c} J_{c}, \operatorname{Tr}\left(J_{a} J_{b}\right)=-(1 / 2) \delta_{a b}$. Note that $\bar{A}$ is not the complex conjugate of $A$.

Let $F^{a}$ and $\bar{F}^{a}$ the Yang-Mills curvatures associated to $A^{a}$ and $\bar{A}^{a}$. From the point of view of the equations of motion, the relation between Chern-Simons theory and general relativity is contained in the fact that the Chern-Simons equations,

$$
F^{a}=0, \quad \bar{F}^{a}=0,
$$

are equivalent to the three-dimensional Einstein equations. Thus, studying the space of solutions of (36) we are studying general relativity.

The 1 -form $A^{a}$ is a $S L(2, C)$ Yang-Mills gauge field because in (29) $x$ is imaginary. For Minkowskian gravity $x=1 / l$ is real and the relevant group is $S O(2,1) \times$ $S O(2,1)$.

\section{Chern-Simons dynamics. Kac-Moody symmetry}

Once we have proved the equality between the Chern-Simons and gravitational actions we can forget about metrics and work with Yang-Mills fields which are much more tractable. We should keep in mind however that the Chern-Simons action is a generalization to general relativity in the sense that it can accept degenerate metrics.

The classical dynamics of Chern-Simons theory is simple to analyse. First, we note that the Chern-Simons action is already in Hamiltonian form. In the $2+1$ decomposition of the gauge field $A^{a}=A_{0}^{a} d t+A_{i}^{a} d x^{i}$, the Chern-Simons action reads,

$$
I\left[A_{i}, A_{0}\right]=\frac{k}{8 \pi} \int d t \int_{\Sigma} \epsilon^{i j} \delta_{a b}\left(A_{i}^{a} \dot{A}_{i}^{b}-A_{0}^{a} F_{i j}^{b}\right)
$$

The coordinates $x^{i}$ are local coordinates on the spatial surface denoted by $\Sigma$. This action has $2 N$ dynamical fields $A_{i}^{a}(a=1, \ldots, N ; i=1,2)$ and $N$ Lagrange multipliers $A_{0}^{a}$. The dynamical fields satisfy the basic equal-time Poisson bracket algebra,

$$
\left\{A_{i}^{a}(x), A_{j}^{b}(y)\right\}=\frac{4 \pi}{k} \epsilon_{i j} \delta^{a b} \delta^{2}(x, y) .
$$

The equation of motion with respect to $A_{0}$ leads to the constraint equation,

$$
\frac{k}{8 \pi} \epsilon^{i j} F_{i j}^{a} \approx 0 \text {. }
$$


which (after properly taken into account boundary condition and boundary terms if the spatial surface has a boundary) generates the gauge transformations $\delta A_{i}^{a}=$ $D_{i} \lambda^{a}$ in the Poisson bracket (38).

Because the equations of motion of Chern-Simons theory are $F=0$ we know that there are no local degrees of freedom in this theory. It is instructive however to count the number of degrees of freedom per point using the Dirac formalism. We have $2 N$ dynamical variables subject to $N$ constrains. These constraints are of first class and generate the $N$ local gauge transformations. Thus the total number of local degrees of freedom is indeed zero. This does not mean that the action is trivial. There are an infinite number of degrees of freedom associated to the breakdown of gauge invariance at the boundary, plus a finite number associated to holonomies along non-contractible loops. Here we shall not consider the holonomies. We refer the reader to $[6,22]$.

The boundary degrees of freedom in Chern-Simons theory can be understood in many different ways. Their existence was first indicated in [49], and Carlip [43,44] first pointed out that they may be responsible for the three-dimensional black hole entropy.

These degrees of freedom are somehow a matter of interpretation rather than a specific calculation. The point is that, at the boundary, is incorrect to identify configurations that differ by a gauge transformation. As discussed in [43] this follows from boundary terms arising in the transformation of the Chern-Simons action under gauge transformations. Alternatively, following [32], one can see that at the boundary the transformations $\delta A_{i}^{a}=D_{i} \lambda^{a}$ are not generated by constraints and therefore they do not represent proper gauge transformations. In summary, the symmetry is still there but its interpretation is different.

This point can be exhibited in the following calculation. This analysis is taken from [50] and [54], with minor modifications. To simplify the notation, let us use differential form notation in the spatial manifold $A=A_{i} d x^{i}$. We rewrite the action (37) in the form,

$$
I\left[A, A_{0}\right]=\frac{k}{4 \pi} \int d t \int_{\Sigma}\left(A_{\wedge} \dot{A}-A_{0} F\right),
$$

where the symbol $\int$ includes the trace Tr. The constraint $F=0$ implies,

$$
A=g^{-1} d g
$$

from where we derive two useful identities,

$$
\delta A=D\left(g^{-1} \delta g\right), \quad \dot{A}=D\left(g^{-1} \dot{g}\right) .
$$

$D$ represents the covariant derivative in the flat connection $A$ given in (41): $D=$ $d+[A$,$] . Our goal is to compute the commutator of two solutions of the form (41).$

Consider a non-canonical Lagrangian of the form $L=l_{a}(z) \dot{z}^{a}$ whose variation reads 


$$
\delta L=\delta z^{a} \sigma_{a b} \dot{z}^{b}, \quad \sigma_{a b}(z)=\partial_{a} l_{b}-\partial_{b} l_{a} .
$$

If $\sigma$ is non-degenerate, the Poisson bracket of $z^{a}$ with itself is given by

$$
\left\{z^{a}, z^{b}\right\}=J^{a b}(z)
$$

where $J$ is the inverse of $\sigma, J^{a b} \sigma_{b c}=\delta_{c}^{a}$. The Jacobi identity for $J$ follows from the Bianchi identity for $\sigma$. If $L=p \dot{q}$ the above construction yields $[q, p]=1$, as expected. Following [54], we shall use this method to compute the Poisson bracket between solutions of the form (41).

The idea is to replace the solution (41) in the action (40) and compute its variation on the surface (41). Since after replacing (41) in (40) only the kinetic term survives the variation of $I$ reads,

$$
\begin{aligned}
\delta I & =-\frac{k}{2 \pi} \int \dot{A} \wedge \delta A, \\
& =-\frac{k}{2 \pi} \int_{\Sigma} D\left(g^{-1} \dot{g}\right)_{\wedge} D\left(g^{-1} \delta g\right), \\
& =\frac{k}{2 \pi} \int_{\partial \Sigma} D_{\varphi}\left(g^{-1} \dot{g}\right) g^{-1} \delta g .
\end{aligned}
$$

The last equality follows from $D_{\wedge} D=F=0$. We thus find that the variation of $I$ on the surface (41) depends only on the boundary values of $g$. This is of course the well known fact that the variation of the $W Z W$ action can be written as a local functional of the boundary. It also means that the only non-trivial degrees of freedom arise at the boundary, and they are the values of $g$ at the boundary. Using (42), the variation of $I$ can be written as,

$$
\delta I=\frac{k}{2 \pi} \int_{\partial \Sigma} \dot{A}_{\varphi} \frac{1}{D_{\varphi}} \delta A_{\varphi}
$$

where $1 / D_{\varphi}$ is the inverse of the operator $D_{\varphi}=\partial_{\varphi}+\left[A_{\varphi},\right]$ which we assume exists (we exclude functions satisfying $D_{\varphi} f=0$ ). Comparing this variation with (43) and (44), we find the Poisson bracket of $A_{\varphi}$ with itself,

$$
\left\{A_{\varphi}, A_{\varphi}\right\}=\frac{2 \pi}{k} D_{\varphi}
$$

where the derivative term in $D_{\varphi}$ should be understood as the derivative of a Dirac delta function. Finally, we make a Fourier expansion,

$$
A(\varphi)=\frac{2}{k} \sum_{n} T_{n}^{a} e^{i n \varphi}
$$

and obtain the quantum commutator $(\hbar=1)$

$$
\left[T_{n}^{a}, T_{m}^{b}\right]=i \epsilon_{c}^{a b} T_{n+m}^{c}+n \frac{k}{2} \delta^{a b} \delta_{n+m}
$$


Some comments are in order here.

(i) It is clear that the equations of motion do not force $A_{\varphi}$ to be zero. Actually, in the sector with chiral boundary conditions $A_{\varphi}$ is arbitrary. On the other hand, $A_{\varphi}$ generates "gauge" transformations acting on itself. Indeed, let $Q(\eta)=(k / 2 \pi) \int \eta_{a} A_{\varphi}^{a}$, it follows directly from (47) that,

$$
\delta A_{\varphi}^{a}=\left[A_{\varphi}^{a}, Q\right]=D_{\varphi} \eta^{a} .
$$

However, here the interpretation is quite different because (50) is not generated by the "Gauss law" constraint $F=0$. Instead, it is generated by $A_{\varphi}$ which is different from zero. The symmetry (50) is a global -not gauge- symmetry. This means that configurations which differ by a transformation of the form (50) are physically distinct. This is the origin of boundary degrees of freedom in Chern-Simons theory.

(ii) We have only computed the bracket between the values of the gauge field $A$, not the group element $g$. This will be enough for our purposes but we remark that the problem of computing the bracket of $g(x)$ with itself leads to interesting constructions which involve quantum groups. Another remarkable application of Chern-Simons theory which we will not consider here is knot theory [49].

(iii) The algebra (49) is known as affine, or Kac-Moody, $S U(2)$ algebra. This algebra is a non-Abelian generalization of the usual Heisenberg algebra $\left[a_{n}, a_{m}\right]=$ $n \delta_{n+m}$. Note that the last term in (49) is precisely the algebra of three oscillators. The first term couples them and, for example, alter the number of degrees of freedom (degeneracy). Unitary representations for (49) are well understood (see, for example, $[47,58,48])$ and they exist provided $k$ is an integer.

(iv) Finally, an exercise for interested students: derive (49) starting from (38) by fixing the gauge $A_{r}=0$, solving the constraints $F=0$ and constructing the Dirac bracket. Note that the constraint $F=0$ is a differential equation which on a manifold with boundary will necessarily lead to an integration function. Identify this function with $A_{\varphi}$ above.

\section{THE BROWN-HENNEAUX CONFORMAL SYMMETRY}

As a final point, we briefly mention one the main application of the affine algebra (49) to three-dimensional gravity. The content of this section follows the original papers $[7,41]$ for the derivation of the conformal algebra, [55-57] for the $S U(2)_{k} \rightarrow$ Virasoro reduction, and $[10,23,45]$ for the statistical interpretation of the conformal algebra. See $[35,40,59]$ for other aspects and further developments.

Let us show how does the Brown-Henneaux conformal algebra for anti-de Sitter spacetimes is derived from (49). We follow [41]. (See [36] for an alternative derivation based on a twisted Sugawara construction, and [46] for a supersymmetric generalization.)

It was pointed out in [41] that the full affine algebra does not represent the dynamics of anti-de Sitter spacetimes. Indeed, computing the metric associated to 
the boundary conditions invariant under (49), one discoveres that they match the boundary conditions found in [7] only if one imposes the additional restrictions [41]

$$
T_{n}^{3}=0, \quad T_{n}^{+}=\delta_{n}^{0},
$$

on the affine generators $\left(T^{ \pm}=T^{1} \pm i T^{2}\right)$.

These reductions conditions were first studied in [55] in the context of twodimensional gravity. It was shown in that reference that the residual algebra leads to a Virasoro algebra with a central charge $c=-6 k$. Starting from (49) this result can be proved as follows. We regard (51) as a set of second class constraints to be imposed in the algebra (49). We then construct the Dirac ${ }^{5}$ bracket [, ]* which is invariant under (51): $\left[T_{n}^{3}, X\right]^{*}=\left[T_{n}^{+}, X\right]^{*}=0$ for all $X$. The only remaining component $T_{n}^{-}$can be renamed as $L_{n}=(1 / k) T_{n}^{-}$and it follows that, in the Dirac bracket, $L_{n}$ satisfies the Virasoro algebra

$$
\left[L_{n}, L_{m}\right]^{*}=(n-m) L_{n+m}+\frac{c}{12} n\left(n^{2}-1\right) \delta_{n+m}
$$

with central charge $c=-6 k$ (see [40] for an explicit calculation). From the value of $k$ given in (33) we find,

$$
c=\frac{3 l}{2 G}
$$

which is the correct Brown-Henneaux central charge [7].

The $c=3 l / 2 G$ Virasoro algebra was discovered in 1986 [7]. However only recently [10] it was pointed out that it plays a central role in the understanding of quantum three-dimensional black holes. The idea is the following.

The first input is that the zero modes $L_{0}$ and $\bar{L}_{0}$ of the Virasoro algebra are related to the mass and spin of anti-de Sitter spacetime as [7],

$$
M l=L_{0}+\bar{L}_{0}-\frac{c}{12}, \quad J=L_{0}-\bar{L}_{0}
$$

For the black hole [9], these two parameters are related to the inner and outer horizon via,

5) For those not familiar with the Dirac bracket formalism, see [53] for a complete treatment. The idea is to find the Poisson bracket acting on a system with constraints. For example, a free particle in three dimensions with a canonical kinetic term $\int p_{i} \dot{q}^{i}$ has the standard Poisson bracket structure. Suppose we decide to restrict the movement of the particle according to $q^{3}=0, p_{3}=0$. The new Poisson bracket is the same as before with the only modification that the coordinates $q^{3}$ and $p_{3}$ are removed. There are cases, however, in which the constraints are complicated functions of the canonical variables and one can not remove the right coordinates just by inspection. Let us consider a system with variables $z^{a}$ and a Poisson bracket $\left[z^{a}, z^{b}\right]=J^{a b}$. Now, we impose the restrictions $\phi_{\alpha}(z)=0$ such that $\operatorname{det} C_{\alpha \beta} \neq 0$ where $C_{\alpha \beta}=\left[\phi_{\alpha}, \phi_{\beta}\right]$. The Dirac bracket $[,]^{*}=[]-,\left[, \chi_{\alpha}\right] C^{\alpha \beta}\left[\chi_{\beta},\right]$ is antisymmetric, satisfies the Jacobi identity and is invariant under the constraints, $\left[X, \phi_{\alpha}\right]^{*}=0$ for all $X$. 


$$
M l=\frac{r_{+}^{2}+r_{-}^{2}}{8 G l}, \quad J=\frac{2 r_{+} r_{-}}{8 G l}
$$

The Virasoro algebra (52) represents a symmetry of the theory, just like the angular momentum algebra, $\left[L_{i}, L_{j}\right]=i \varepsilon_{i j k} L_{k}$, is the symmetry algebra of a rotational invariant Lagrangian. Suppose that the algebra (52) is the symmetry algebra associated to some conformal field theory which is unitary $\left(L_{0}, \bar{L}_{0} \geq 0\right)$ and modular invariant. Modular invariance implies that the partition function,

$$
Z[\tau]=\operatorname{Tr} e^{2 \pi i \tau\left(L_{0}-c / 24\right)-2 \pi i \bar{\tau}\left(\bar{L}_{0}-c / 24\right)},
$$

satisfies

$$
Z\left[\tau^{\prime}\right]=Z[\tau], \quad \tau^{\prime}=\frac{a \tau+b}{c \tau+d},
$$

for any $a, b, c, d \in Z$ and $a d-b c=1$. The parameter $\tau$ is the modular parameter, or complex structure, of the torus on which the CFT is defined. We recall that the partition function (56) has a precise interpretation in the black hole manifold. The Euclidean black hole has the topology of a solid torus whose modular parameter is given in (19) and $L_{0}+\bar{L}_{0}$ is the Hamiltonian of the theory (up to an additive constant that we discuss below). This is actually implicit in (54).

Since $Z[\tau]$ is modular invariant, we can evaluate $Z[-1 / \tau]$ in the limit $\operatorname{Im}(\tau) \rightarrow 0$. Assuming $L_{0}, \bar{L}_{0} \geq 0$ we obtain,

$$
Z[\tau] \sim\left|\exp \left(\frac{2 \pi i c}{24 \tau}\right)\right|^{2}
$$

From (19), $\tau=i \beta / 2 \pi l$ (non-rotating case), and (53) we find exactly the semiclassical Gibbons-Hawking limit (20). (Exercise: generalize this to the rotating case.) This is the canonical $[9,37,38,52]$ version of the results obtained in [10]. Note that the limit $\operatorname{Im}(\tau) \rightarrow 0$ corresponds to small $\beta$ and, according to (18), large values of $M$. This is a characteristic of the three dimensional black hole not shared by the Schwarzschild solution. The temperature in three dimensions decreases with the mass, the specific heat is positive and the canonical ensemble is well-defined.

A microcanonical calculation follows by writing the partition function (56) in the form,

$$
Z[\tau]=\sum_{L_{0}, \bar{L}_{0}} \rho\left(L_{0}, \bar{L}_{0}\right) e^{2 \pi i \tau\left(L_{0}-c / 24\right)-2 \pi i \bar{\tau}\left(\bar{L}_{0}-c / 24\right)}
$$

where $\rho\left(L_{0}, \bar{L}_{0}\right)$ is the number of states with eigenvalues $L_{0}, \bar{L}_{0}$. Using the approximation (58) in (59) one can extract the number of states $\rho\left(L_{0}, \bar{L}_{0}\right)$ by a contour integral obtaining,

$$
\rho\left(L_{0}, \bar{L}_{0}\right) \sim e^{2 \pi \sqrt{c L_{0} / 6}+2 \pi c \sqrt{c \bar{L}_{0} / 6}} .
$$


This is known as Cardy formula. It is amusing to check that using (53), (54) and (55), the associated entropy is exactly equal to the Bekenstein-Hawking value $S=A / 4 G$ with $A=2 \pi r_{+}[10]$.

Actually, the above calculation is true provided the black hole mass is large enough: $M l>>c / 12$ (see (54)). The shift $-c / 12$ appearing in (54) (which should be written as $-c / 24-c / 24)$ is the source of a number of issues. For unitary theories, on which the above calculation makes sense, it means that the mass spectrum is $M \geq-c / 12$ and thus not only black holes enter in the partition function but also the conical singularities (particle solutions) introduced in [8]. Curiously when writing canonical expressions for the Virasoro generators, either using the Liouville approach [41] or the twisted Sugawara operator [36], one finds $M \geq 0$. This looks fine because the entropy should be associated to black holes spacetimes having horizons and not to the particle solutions. However, if one restricts the spectrum to positive masses, then the saddle point approximation (58) is not valid. In summary, the CFT whose symmetry is generated by (52), and that we assumed existed, does not seem to be related to general relativity.

We shall end here. See [45,11] for discussions on this last point, [59] for a proposal to resolve this problem within general relativity, and $[11,13,14]$ for the string theory side of it.

\section{ACKNOWLEDGMENTS}

The author would like to thank the organizers of the VIII Mexican School on Particles and Fields for the kind invitation to deliver these lectures. I would also like to thank S. Carlip, M. Henneaux, M.Ortiz and A.Ritz for many conversations and correspondence which had been very helpful to understand the ideas presented here. Financial support from CICYT (Spain) grant AEN-97-1680, and the Spanish postdoctoral program of Ministerio de Educación y Ciencia is also acknowledged.

\section{REFERENCES}

1. A. Strominger and C. Vafa, Phys. Lett. B379, 99 (1996)

2. C.G. Callan and J. Maldacena, Nucl.Phys. B472, 591-610 (1996).

3. C. Rovelli, Phys. Rev. Lett 77, 3288 (1996).

4. A. Ashtekar, J. Baez, A. Corichi, K. Krasnov, Phys. Rev. Lett 80, 904 (1998).

5. A. Achúcarro and P.K. Townsend, Phys. Lett. B180, 89 (1986).

6. E. Witten, Nucl. Phys. B 311, 46 (1988).

7. J.D. Brown and M. Henneaux, Commun. Math. Phys. 104, 207 (1986).

8. S. Deser, R. Jackiw and G. 't Hooft, Ann. Phys. 152, 220 (1984); S. Deser and R. Jackiw, Ann. Phys. (NY), 153, 405 (1984)

9. M. Bañados, C. Teitelboim and J.Zanelli, Phys. Rev. Lett. 69, 1849 (1992); M. Bañados, M. Henneaux, C. Teitelboim and J.Zanelli, Phys. Rev. D48, 1506 (1993).

10. A. Strominger, High Energy Phys. 02009 (1998). 
11. E. Martinec, "Conformal field theory, geometry, and entropy", hep-th/9809021

12. M. Henningson, K. Skenderis, J.High Energy Phys 9807, 023 (1998).

13. A. Giveon, D. Kutasov and N. Seiberg, "Comments on string theory on $\operatorname{adS}(3)$ ", e-Print Archive: hep-th/9806194

14. J. de Boer, H. Ooguri, H. Robins and J. Tannenhauser, "String theory on $\operatorname{adS}_{3}$, hep-th/9812046

15. K. Skenderis, "Black holes and branes in string theory", hep-th/9901050.

16. S. Carlip, Quantum Gravity in 2+1 Dimensions, Cambridge University Press (1998).

17. G. Gibbons and S.W. Hawking, Phys.Rev. D15, 2752 (1977)

18. G. Gibbons, Phys.Lett. A61, 3 (1977).

19. J. Maldacena, Adv. Theor. Math. Phys. 2, 231 (1998).

20. S.S. Gubser, I.R. Klebanov, A.M. Polyakov, Phys. Lett. B428, 105 (1998)

21. E. Witten, Adv. Theor. Math. Phys. 2253 (1998)

22. J.E. Nelson and T. Regge, Nucl.Phys.B328, 190 (1989); Phys.Lett. B272, 213 (1991); Commun. Math. Phys. 141, 211 (1991).

23. D. Birmingham, I. Sachs and A. Sen, Phys.Lett. B424, 275-280 (1998)

24. D.N. Page, "Black hole information", hep-th/9305040

25. S.W. Hawking and G.F.R. Ellis (1973), The Large Scale Structure of Space-time, Cambridge University Press.

26. S.W.Hawking, Nature (London) 248, 30 (1974).

27. G. Howowitz, "The Dark Side of String Theory: Black Holes and Black Strings", hep-th/9210119.

28. M. Bañados, C. Teitelboim and J.Zanelli, Phys. Rev. D49, 975 (1994).

29. N.D. Birrell and P.C.W. Davies, "Quantum Fields in curved space time", Cambridge University Press (1982).

30. R.M. Wald (1984), General Relativity University Press, Chicago, USA.

31. G.T. Horowitz, "The origin of black hole entropy in string theory", gr-qc/9604051.

32. T. Regge and C. Teitelboim, Ann. Phys. (N.Y.) 88, 286 (1974).

33. J.D.Brown, E.A.Martinez and J.W.York, Phy. Rev. Lett. 66, 2281 (1991)

34. M. Bañados, C. Teitelboim and J.Zanelli, Phys. Rev. Lett. 72, 957 (1994).

35. P. Navarro and J. Navarro-Salas, Phys. Lett. B439, 262 (1998).

36. M. Bañados, Phys. Rev. D52, 5816 (1995).

37. M. Bañados, T. Brotz and M. Ortiz, "Boundary dynamics and the statistical mechanics of the $2+1$ dimensional black hole", hep-th/9802076, To appear in Nucl.Phys.B

38. J. Maldacena, A. Strominger, "adS(3) black holes and a stringy exclusion principle". e-Print Archive: hep-th/9804085

39. T. Brotz, M. Ortiz and A. Ritz, "On Modular Invariance and 3D Gravitational Instantons", hep-th/9903222.

40. M. Bañados, "Three dimensional quantum geometry and black holes", hepth/9901148

41. O. Coussaert, M. Henneaux, P. van Driel, Class.Quant.Grav. 12, 2961 (1995).

42. S. Carlip, C. Teitelboim, Phys.Rev. D51, 622 (1995).

43. S. Carlip, Phys. Rev. D51, 632 (1995)

44. S. Carlip, Phys. Rev. D55, 878, (1997)

45. S. Carlip, Class. Quant. Grav. 153609 (1998) 
46. M. Bañados, K. Bautier, O. Coussaert, M. Henneaux and M. Ortiz, Phys. Rev. D58 085020 (1998)

47. D. Gepner and E. Witten, Nucl.Phys. B278, 493 (1986).

48. P. Di Francesco, P. Mathieu, D. Senechal. "Conformal field theory" New York, USA: Springer (1997) $890 \mathrm{p}$.

49. E. Witten, Commun. Math. Phys. 121, 351 (1989).

50. G. Moore and N. Seiberg, Phys.Lett. B220, 422 (1989); S. Elitzur, G. Moore, A. Schwimmer and N. Seiberg, Nucl. Phys. B326, 108 (1989)

51. P. Goddard, A. Kent and D. Olive, Comm. Math.Phys. 103, 105, (1986).

52. M. Bañados, F. Méndez Phys. Rev. D58 104014 (1998)

53. M. Henneaux and C. Teitelboim, Quantization of Gauge Systems (Princeton University Press, Princeton, 1992).

54. E. Witten, Commun. Math. Phys. 92, 455 (1984).

55. A.M. Polyakov, Int. J. Mod. Phys. A5 (1990) 833.

56. A. Alekseev and S. Shatashvili, Nucl. Phys. B323, 719 (1989).

57. P. Forgács, A. Wipf, J. Balog, L. Fehér and L. O'Raifeartaigh, Phys. Lett. 227 B (1989) 214.

58. P. Goddard and D. Olive, Int. Journ. Mod. Phys. A1, 303, (1986).

59. M. Bañados, "Twisted sectors in three dimensional gravity", hep-th/9903178 